\title{
Three-dimensional instability in flow over a backward-facing step
}

\author{
By DWIGHT BARKLEY ${ }^{1}$, M. GABRIELA M. GOMES \\ AND RONALD D. HENDERSON ${ }^{2}$ \\ ${ }^{1}$ Mathematics Institute, University of Warwick, Coventry CV4 7AL, UK \\ ${ }^{2}$ Aeronautics and Applied Mathematics, California Institute of Technology, \\ Pasadena, CA 91125, USA
}

(Received 10 April 2002 and in revised form 1 July 2002)

Results are reported from a three-dimensional computational stability analysis of flow over a backward-facing step with an expansion ratio (outlet to inlet height) of 2 at Reynolds numbers between 450 and 1050. The analysis shows that the first absolute linear instability of the steady two-dimensional flow is a steady three-dimensional bifurcation at a critical Reynolds number of 748. The critical eigenmode is localized to the primary separation bubble and has a flat roll structure with a spanwise wavelength of 6.9 step heights. The system is further shown to be absolutely stable to two-dimensional perturbations up to a Reynolds number of 1500 . Stability spectra and visualizations of the global modes of the system are presented for representative Reynolds numbers.

\section{Introduction}

The separated flow generated as fluid passes over a backward-facing step is of interest for a variety of reasons. First, separated flows produced by an abrupt change in geometry are of great importance in many engineering applications. This has driven numerous studies of the flow over a backward-facing step during the past 30 years (e.g. Goldstein et al. 1970; Denham \& Patrick 1974; Armaly et al. 1983; Adams \& Johnston 1988). Also, the backward-facing step is an extreme example of separated flows that occur in aerodynamic devices such as high-lift airfoils at large angles of attack. In these flows separation may be created by a strong adverse pressure gradient rather than a geometric perturbation, but the flow topology is similar. Secondly, from a fundamental perspective, there is a strong interest in understanding instability and transition to turbulence in non-parallel open flows. Transition mechanisms in parallel flows such as plane channels and pipes have received substantial attention (e.g. Bayly, Oszag \& Herbert 1988 and references therein; Butler \& Farrell 1993; Hamilton, Kim $\&$ Waleffe 1995). While many questions remain, these basic flows are understood considerably better than the non-parallel flows arising in more complex geometries. In this context the flow over a backward-facing step has emerged as a prototype of a non-trivial yet simple geometry in which to examine the onset of turbulence (Avva 1988; Kaiktsis, Karniadakis \& Orszag 1991, 1996; Akselvoll \& Moin 1993; Le, Moin \& Kim 1997). Finally, from a strictly computational perspective, the steady two-dimensional flow over a backward-facing step is an established benchmark in computational fluid dynamics (e.g. Ghia, Osswald \& Ghia 1989; Gartling 1990). New computational studies such as the highly accurate stability computations presented here will expand the database for this benchmark problem. 
The two-dimensional, absolute, linear stability of this flow has been examined extensively and is discussed in several publications (Gartling 1990; Gresho et al. 1993; Fortin et al. 1997). Computational studies have established that the two-dimensional laminar flow is linearly stable with respect to two-dimensional perturbations up to a Reynolds number of at least $R e=600$. (As discussed in $\S 2$, several definitions of the Reynolds number are used in the literature. Appropriate reference scales for this problem are the upstream centreline velocity $U_{\infty}$ and step height $h$; all cited results are expressed in these units.) However, additional computational evidence supports the existence of a local convective instability (again to two-dimensional disturbances) for a sizable portion of the domain at Reynolds numbers above 525 (Kaiktsis et al. 1996).

Denham \& Patrick (1974) conducted experiments on laminar flow over a backwardfacing step with an expansion ratio of 3 . Velocity profiles and reattachment lengths of the primary recirculation zone were measured in the steady two-dimensional regime for Reynolds numbers up to 344. Denham \& Patrick also describe transient three-dimensional flows within the primary recirculation zone following perturbations (obtained by tapping the channel) at $R e=344$. Experiments by Armaly et al. (1983) on air flow in a backward-facing step geometry with nominal expansion ratio of approximately 2 provide quantitative measurements of two- and three-dimensional flows over a large range of Reynolds number, from about 50 to 6000. In addition to providing data on separation and reattachment points, streamwise velocity measurements are reported for several Reynolds numbers throughout the range of the study. Below Reynolds number 300 the flow is essentially spanwise invariant, although some deviation from two-dimensionality necessarily exists near the lateral sidewalls of the channel (see below). Above $R e=300$ there is a measurable deviation from twodimensionality. At about the same Reynolds number a secondary separation bubble is observed on the upper wall of the channel (the wall opposite the step).

Ghia et al. (1989) computed two-dimensional solutions of the backward-facing step flow throughout the laminar regime and found good agreement with the twodimensional flows observed by Armaly et al. (1983). They discuss two mechanisms for the onset of three-dimensionality based on available information. They postulate that instability of the two-dimensional flow could result from a Taylor-Görtler instability after the formation of the secondary separation bubble on the upper wall because the main flow is then subject to destabilizing concave curvature. They also consider, but reject, the possibility that the sidewall boundary layer contributes to three-dimensional transition. Kaiktsis et al. (1991) studied the onset of three-dimensionality using direct numerical simulations, primarily for the case of a spanwise-periodic domain. They reported both two- and three-dimensional instability (unsteadiness) at approximately the same Reynolds number: $R e \simeq 525$. However, Kaiktsis et al. (1996) later showed that this two-dimensional instability was convective rather than absolute. They report that the three-dimensional instability occurs at the 'boundaries between the primary and secondary recirculation zones with the main channel flow'.

Williams \& Baker (1997) have conducted three-dimensional simulations in a domain with lateral sidewalls having the same expansion and aspect ratio (spanwise length $L_{z}$ to step height $h$ ) used by Armaly et al. (1983), and they reproduce the laminar three-dimensional flows observed experimentally. They find deviations from two-dimensionality near the sidewalls at Reynolds numbers where Armaly et al. (1983) report two-dimensional flows and note that 'the transition from two- to threedimensional flow is not an abrupt change but rather a continuous penetration of the three-dimensional flow, fed by a wall jet, from the sidewall to the central symmetry 
plane'. The implication is that the three-dimensionality observed in the experiments results from an extrinsic effect induced by the lateral boundary conditions, and thus will probably depend on the system aspect ratio. The observed three-dimensionality does not follow from a fundamental hydrodynamic instability of a two-dimensional flow.

Thus in spite of the numerous investigations of flow over a backward-facing step available in the literature, some of the most basic questions for this flow remain open: in the ideal problem with no sidewalls, at what Reynolds number does the flow first become linearly unstable, what is the nature of this instability, and where in the flow does it originate? These are the questions we wish to address. We focus on the accurate determination of the initial intrinsic three-dimensional instability. This is similar in spirit to the work of Kaiktsis et al. (1991) which attempted to quantify the transition to three-dimensionality in backward-facing step flow via direct numerical simulations. Our approach is a computational bifurcation analysis of the flow. We will show that the primary instability for the backward step flow is a three-dimensional, steady bifurcation.

Our presentation is organized as follows. In $\S \S 2$ and 3 we formulate the problem and describe our numerical methods. In $\S 4$ we report the results of our stability analysis for Reynolds numbers up to 1050 , and in $\S 5$ we discuss these results in the context of experiments and transition to turbulence for the flow over a backward-facing step.

\section{Problem formulation}

Consider the motion of a viscous fluid contained between two fixed plates with a step change in separation distance at the origin. We take the flow direction to be such that fluid moves toward the larger gap, i.e. a backward-facing step. The fluid is assumed to have constant density $\rho$ and constant kinematic viscosity $v$. The Reynolds number is $R e \equiv U_{\text {ref }} L_{\text {ref }} / v$, where the reference scales $L_{\text {ref }}$ and $U_{\text {ref }}$ for length and velocity are specified below.

The fluid motion is governed by the incompressible Navier-Stokes equations, written in non-dimensional form as

$$
\begin{gathered}
\frac{\partial \boldsymbol{u}}{\partial t}=\boldsymbol{N}(\boldsymbol{u})-\nabla p+\frac{1}{R e} \nabla^{2} \boldsymbol{u} \text { in } \Omega, \\
\nabla \cdot \boldsymbol{u}=0 \quad \text { in } \Omega,
\end{gathered}
$$

where $\boldsymbol{u}(\boldsymbol{x}, t)$ is the velocity field, $p(\boldsymbol{x}, t)$ is the static pressure, $\Omega$ is the computational domain, and $N(\boldsymbol{u})$ represents the nonlinear advection term:

$$
N(\boldsymbol{u}) \equiv-(\boldsymbol{u} \cdot \nabla) \boldsymbol{u} .
$$

Figure 1 illustrates the computational domain under consideration and also serves to define the geometric parameters for the problem. We consider a step of height $h$ and take the edge of the step as the origin of our coordinate system. Fluid arrives from an inlet channel of height $\alpha h$ and flows downstream into an outlet channel of height $(1+\alpha) h$. In this study we fix $\alpha=1$, giving an expansion ratio (outlet to inlet) of $1+1 / \alpha=2$. The inflow and outflow lengths $L_{i}$ and $L_{o}$ should be large enough that the results are independent of these parameters. At the inlet, $L_{i}=h$ is sufficient for the range of Reynolds numbers we consider (Kaiktsis et al. 1991; Williams \& Baker 1997). The required outflow length $L_{o}$ varies with Reynolds number. As part of our convergence study (see $\$ 3.3 .1$ below) we found acceptable values to lie in the 


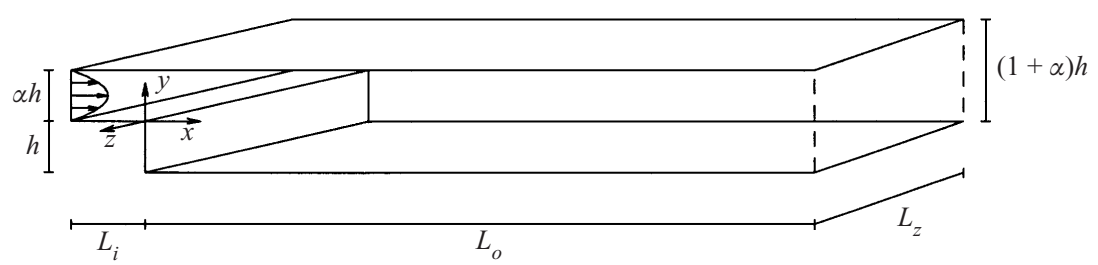

FIGURE 1. Flow geometry for the backward-facing step. The origin of the coordinate system is at the step edge. In this work we take the ratio of inlet height to step height as $\alpha=1$, so that the expansion ratio is $1+1 / \alpha=2$.

range $15 h \leqslant L_{o} \leqslant 55 h$. Finally we take the system to be homogeneous in the spanwise direction and we take the system to be infinitely large in this direction, i.e. $L_{z}=\infty$, by considering all spanwise Fourier modes (see $\S 3$ ).

Boundary conditions are imposed on the flow as follows. At the inflow boundary $\left(x=-L_{i}, 0 \leqslant y \leqslant \alpha h\right)$ we impose a parabolic profile: $u=4 y(\alpha h-y) /(\alpha h)^{2}, v=w=0$. Along the step and all channel walls we impose no-slip conditions. At the outflow boundary $\left(x=L_{o},-h \leqslant y \leqslant \alpha h\right)$ we impose a standard outflow boundary condition for velocity and pressure:

$$
\partial_{x} \boldsymbol{u}(\boldsymbol{x}, t)=(0,0,0), \quad p(\boldsymbol{x}, t)=0 .
$$

Along all other boundaries the pressure is forced to satisfy the high-order Neumann boundary condition given by Karniadakis, Israeli \& Orszag (1991).

Several different choices of non-dimensionalization appear in the literature. Table 1 provides a representative list of reference scales, $L_{\text {ref }}$ and $U_{\text {ref }}$, and the corresponding Reynolds number in the case $\alpha=1$. The step height $h$ is a natural length scale for defining the problem geometry and measuring quantities like downstream separation points, and it is the most common choice for $L_{\text {ref }}$. Other common length scales used are the downstream channel height $(1+\alpha) h$ or, if the incoming flow is turbulent, the momentum thickness of the upstream boundary layer. Two different velocity scales are commonly used: the maximum upstream centreline velocity $U_{\infty}$ and the average upstream velocity $\bar{U}$ :

$$
\bar{U}=\frac{1}{\alpha h} \int_{0}^{\alpha h} u(y) \mathrm{d} y .
$$

Note that $\bar{U}=(2 / 3) U_{\infty}$ for parabolic inflow velocity. In the present work we take $L_{\text {ref }}=h$ and $U_{\text {ref }}=U_{\infty}$, giving the Reynolds number as

$$
R e \equiv \frac{U_{\infty} h}{v} .
$$

This definition of Reynolds number is independent of $\alpha$. All quantities cited from the literature (Reynolds numbers, separation points, velocities, eigenvalues, etc.) are rescaled using this non-dimensionalization.

\section{Computational methods}

Our computations consist of two parts. First, we obtain steady two-dimensional solutions from either time-dependent simulations or Newton methods. Second, we determine relevant bifurcation points along the steady branch of solutions via two- and three-dimensional linear stability analysis. The method follows that used previously 


$\begin{array}{lccc}\text { Authors } & L_{\text {ref }} & U_{\text {ref }} & R e_{\alpha=1} \\ \text { Denham \& Patrick (1974) } & h & (2 / 3) U_{\infty} & (2 / 3) U_{\infty} h / v \\ \text { Armaly et al. }(1983) & 2 \alpha h & (2 / 3) U_{\infty} & (4 / 3) U_{\infty} h / v \\ \text { Kaiktsis } \text { et al. }(1991) & h & (4 / 3) U_{\infty} & (4 / 3) U_{\infty} h / v \\ \text { Gresho et al. }(1993) & (1+\alpha) h & (2 / 3) U_{\infty} & (4 / 3) U_{\infty} h / v \\ \text { Adams \& Johnston (1988) } & h & U_{\infty} & U_{\infty} h / v \\ \text { Present study } & h & U_{\infty} & U_{\infty} h / v\end{array}$

TABLE 1. Comparison of reference scales used in various studies. The most common choice is the last one: $L_{\text {ref }}=h$ (step height) and $U_{\text {ref }}=U_{\infty}$ (upstream centreline velocity). Different scalings lead to different definitions of the Reynolds number, tabulated for $\alpha=1$.

by Mamun \& Tuckerman (1995), Barkley \& Henderson (1996), and Barkley \& Tuckerman (1999).

All of the calculations were carried out using a non-conforming spectral element program (Prism, Henderson 1994). In the spectral element method a two-dimensional domain $\Omega$ is represented by a mesh of $K$ elements. Within each element both the geometry and the solution variables, in this case $\boldsymbol{u}(\boldsymbol{x}, t)$ and $p(\boldsymbol{x}, t)$, are represented using $N$ th-order (Legendre) polynomial expansions. Figure 2 shows the basic computational domains used for simulations of the backward-facing step flow over the entire range of Reynolds numbers. Non-conforming elements allow local mesh refinement in regions like the step corner yet preserve the block structure of the calculations. Computational domains with various refinement levels and outflow lengths were used at different Reynolds numbers and will be discussed in $\$ 3.3 .1$ below. Beyond the details of the polynomial basis and the treatment of non-conforming elements in the mesh, the method follows a standard Galerkin finite element procedure to discretize equation (2.1). Henderson \& Karniadakis (1995) discuss further details of the method and solution techniques, along with various validation studies for the particular code employed here.

For the most part the method we use to study bifurcation problems does not depend on any particular spatial discretization so we can describe the relevant algorithms in the following abstract way. Let $u(t)$ be the $n$-dimensional vector containing the discrete representation of the velocity field $\boldsymbol{u}(\boldsymbol{x}, t)$. Discretizing the Navier-Stokes equations (2.1) gives a system of differential algebraic equations schematically of the form

$$
\frac{\mathrm{d} u}{\mathrm{~d} t}=N(u)+L u,
$$

where $L$ and $N(\cdot)$ are linear and nonlinear operators respectively. For linear stability and steady-state calculations we also require equations describing the linear evolution about some given reference state $U$. These equations take the form

$$
\frac{\mathrm{d} u}{\mathrm{~d} t}=\left(N_{U}+L\right) u
$$

where $N_{U}$ is the linearization (Jacobian) of the operator $N$ about state $U$. More specifically, let $\boldsymbol{U}+\boldsymbol{u}$ be an infinitesimal perturbation to a steady flow $\boldsymbol{U}$. Equations for the evolution of $\boldsymbol{u}$ are obtained by replacing the nonlinear advection term in the Navier-Stokes equations with the linearization

$$
\boldsymbol{N}_{\boldsymbol{U}}(\boldsymbol{u}) \equiv-(\boldsymbol{u} \cdot \nabla) \boldsymbol{U}-(\boldsymbol{U} \cdot \nabla) \boldsymbol{u} .
$$




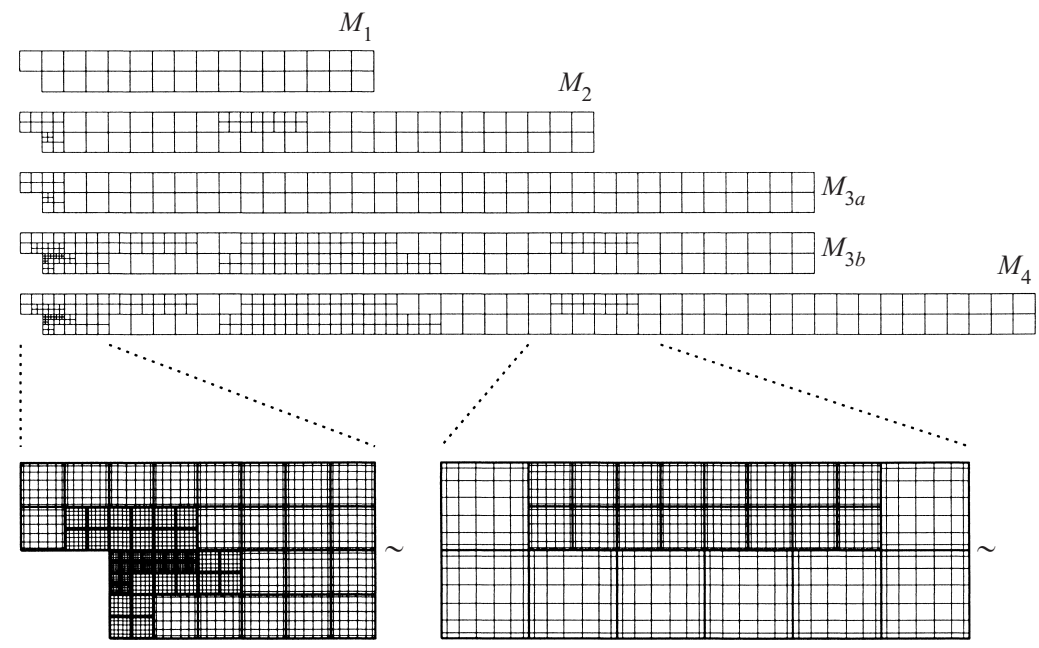

FiguRE 2. Computational domains used in the present study. Subscripts label external dimensions (specifically outflow length $L_{o}$ ). Each domain is divided into $K$ elements. Where necessary, lower-case letters label the degree of internal mesh refinement: the $M_{3 a}$ mesh has $K=83$ elements and the $M_{3 b}$ mesh has $K=203$ elements. Within each element the solution and geometry are represented by $N^{2}$ polynomial coefficients. Two subsections of mesh $M_{4}$ are expanded to show the internal distribution of quadrature points for polynomial order $N=7$. To simulate a three-dimensional flow the solution is decomposed into $M$ Fourier modes in the periodic spanwise direction, each computed on the same two-dimensional grid.

The boundary conditions for the perturbation $\boldsymbol{u}$ are the same as those for the base flow $\boldsymbol{U}$ except that $\boldsymbol{u}=0$ at the inlet. Therefore $\boldsymbol{U}+\boldsymbol{u}$ satisfies the same boundary conditions as $\boldsymbol{U}$.

Our primary tool is a method for evolving some given state forward in time. Define the operators $A$ and $A_{U}$ as follows:

$$
\begin{gathered}
A u(t) \equiv u(t)+\int_{t}^{t+T}(N(u)+L u) \mathrm{d} t^{\prime}, \\
A_{U} u(t) \equiv u(t)+\int_{t}^{t+T}\left(N_{U}+L\right) u \mathrm{~d} t^{\prime} .
\end{gathered}
$$

$A$ gives the nonlinear evolution of $u(t)$ over time interval $T$. It also represents our simulation code as a black box for integrating the Navier-Stokes equations: given a velocity field $u(t)$, it provides the solution at a later time $u(t+T)=A u(t)$. The operator $A_{U}$ gives the analogous linear evolution of $u(t)$ about some given reference state $U$. In practice both equations (3.1) and (3.2) are integrated using the thirdorder semi-implicit splitting scheme described by Karniadakis et al. (1991). For the remainder of this section, however, we will only refer to the operators $A$ and $A_{U}$ rather than the time-dependent Navier-Stokes equations that they represent.

\subsection{Steady-state calculations}

A steady-state or time-independent solution $U$ of (3.1) satisfies $U=A U$ or $(A-I) U=$ 0 , where $I$ is the $n \times n$ identity matrix. Since $A$ is nonlinear and $n$ is large, this equation must be solved iteratively. Let $U_{k} \equiv U(t+k T)$ be a sequence of states that evolve from the initial condition $U_{0}$. The simplest method for finding the steady-state solution $U$ 
is to use fixed-point iteration:

$$
U_{k+1}=A U_{k} .
$$

If all eigenvalues $\mu_{i}$ of $A_{U}$ satisfy $\left|\mu_{i}\right|<1$, then the steady-state solution $U$ is linearly stable and the sequence converges, $U_{k} \rightarrow U$, for most initial conditions. This is equivalent to performing time-integration. To accelerate convergence, once the initial (fast exponential) transients decay we switch to a Newton iteration:

$$
\left.\begin{array}{c}
\left(A_{U_{k}}-I\right) u_{k}=(A-I) U_{k}, \\
U_{k+1}=U_{k}-u_{k} .
\end{array}\right\}
$$

Each Newton iteration requires an inversion of the linear operator $\left(A_{U}-I\right)$. This is accomplished with a generalized minimum residual (GMRES) iterative method (Saad \& Schultz 1986).

For moderate Reynolds numbers (up to $R e \approx 800$ ) our steady-state calculations for the backward-facing step flow consist of only a few explicit steps followed by Newton iterations. For the definition of the operators $A$ and $A_{U}$ we use $T$ equal to a typical time step $\left(\Delta t=5 \times 10^{-3}\right)$. For larger values of the Reynolds number $(R e>800)$ we typically find that the number of GMRES iterations necessary to invert $\left(A_{U}-I\right)$ becomes so large that simple fixed-point iteration (3.5) requires less computation time. The Stokes preconditioning method of Mamun \& Tuckerman (1995) does not work in our case because our numerical operators derive from a third-order splitting scheme (Karniadakis et al. 1991) which does not impose exact incompressibility of the flow. The divergence of the velocity field is of order $\Delta t^{3}$, which is acceptable in direct simulations or in our Newton's method with $\Delta t$ small. However, in Mamun-Tuckerman preconditioning $\Delta t$ is large and so the divergence of the flow becomes very large and the numerical method fails. What this implies for the present study is that our method for computing steady states works well up to and slightly above the Reynolds number for the primary three-dimensional instability of the flow (§4.1). However our method is not well suited for larger Reynolds numbers, for example those which must be attained for two-dimensional linear instability of the flow $(\S 4.3)$. We are able to obtain steady two-dimensional flows between Reynolds number 800 and 1500 only by fixed-point iteration (3.5), effectively time integration. This is feasible since the flows we consider are globally two-dimensionally stable, but the convergence is very slow due to the smallness of the leading two-dimensional eigenvalues $(\S 4.3)$.

\subsection{Stability analysis}

For the linear stability calculations we need to solve the eigenvalue problem $A_{U} \tilde{u}=\mu \tilde{u}$, where $\mu$ is an eigenvalue of the operator $A_{U}$ and $\tilde{u}$ is the corresponding eigenmode. For a time-independent base flow $U$, the eigenvalues $\sigma+\mathrm{i} \omega$ of the linearized NavierStokes operator $N_{U}+L$ are related to the eigenvalues $\mu$ via $\mu=\exp ((\sigma+\mathrm{i} \omega) T)$. An instability occurs if $\sigma>0$ and the resulting bifurcation may be either steady $(\omega=0)$ or oscillatory $(\omega \neq 0)$.

Again we turn to iterative methods. Let $u_{1}$ be some initial guess for the dominant eigenmode of $A_{U}$. To begin we generate the Krylov sequence

$$
u_{1}, \quad u_{2}=A_{U} u_{1}, \quad u_{3}=A_{U} u_{2}, \ldots, \quad u_{m}=A_{U} u_{m-1} .
$$

These vectors form a Krylov subspace that can be represented as the $n \times m$ matrix 
$X=\left\langle u_{1}, u_{2} \ldots u_{m}\right\rangle$. Next we execute the block power iteration

$$
X_{k+1}=A_{U} X_{k} .
$$

Because $X_{k}$ is a Krylov subspace, each iteration of (3.7) requires only a single matrix-vector multiplication to generate the next element of the sequence. Computing an orthonormal basis for the space $X_{k}$ after each iteration gives estimates for the dominant eigenvalues and eigenmodes of $A_{U}$. Any absolute global instability will necessarily be found by this method as long as $u_{1}$ is not exactly orthogonal to the unstable mode of the system. Not only is this very unlikely (and essentially impossible in the presence of random round-off errors), but in addition we often effectively generate several different choices for $u_{1}$ at the same Reynolds number by using different meshes.

For the backward-facing step problem we use a subspace dimension $m$ typically between 20 and 80 and obtain highly accurate leading eigenvalues after $O(100)$ subspace iterations. Typically 200 subspace iterations are required to obtain the four leading eigenvalues accurately. In defining the operator $A_{U}$ for these calculations we use an evolution time of $T=5$. For more details and additional applications see Edwards et al. (1994), Mamun \& Tuckerman (1995), Schatz, Barkley \& Swinney (1995), and Barkley \& Henderson (1996).

Our primary concern here is the three-dimensional stability of steady two-dimensional flows, and in this case the eigenmodes take a special form. Because the system is homogeneous in the spanwise direction, we can decompose general perturbations into Fourier modes with spanwise wavenumbers $\beta$ :

$$
(\boldsymbol{u}, p)=\int_{-\infty}^{\infty}(\hat{\boldsymbol{u}}, \hat{p}) \mathrm{e}^{\mathrm{i} \beta z} \mathrm{~d} \beta .
$$

At linear order, modes with different $|\beta|$ are decoupled. It follows directly from the linearized Navier-Stokes equations that any eigenmode of $A_{U}$ with a given $\beta$ must be of the following form:

$$
\left.\begin{array}{l}
\tilde{\boldsymbol{u}}(x, y, z)=(\hat{u}(x, y) \cos \beta z, \hat{v}(x, y) \cos \beta z, \hat{w}(x, y) \sin \beta z), \\
\tilde{p}(x, y, z)=\hat{p}(x, y) \cos \beta z,
\end{array}\right\}
$$

or an equivalent form obtained by translation in $z$. Restricting attention to modes with a particular $\beta$ reduces the full three-dimensional stability problem at any given Reynolds number to a one-parameter family of problems for the Fourier components $(\hat{u}, \hat{v}, \hat{w})$ of the eigenmode $\tilde{\boldsymbol{u}}(x, y, z)$. These Fourier components are computed on a two-dimensional domain with $\beta$ appearing in the linearized equations as an additional parameter. Linearizing the equations in this way also frees us from the erroneous effects of imposing periodic boundary conditions with an incorrect length, since $\beta$ can be varied continuously. Our stability calculations therefore produce a family of eigenvalues $\mu(\beta)$, or equivalently $\sigma(\beta)+\mathrm{i} \omega(\beta)$, for a discrete set of fixed Reynolds numbers.

As a final note, the steady flows and eigenmodes can be computed on different grids and with different levels of refinement, i.e. a given base flow $U$ can always be interpolated onto an appropriate mesh for the stability calculations. In practice we use the same domains for both the steady flow and stability computations, but frequently use different polynomial orders. In presenting results we use the notation $N_{b}$ and $N_{e}$ to denote the polynomial order used in the base flow and eigenvalue computations. 


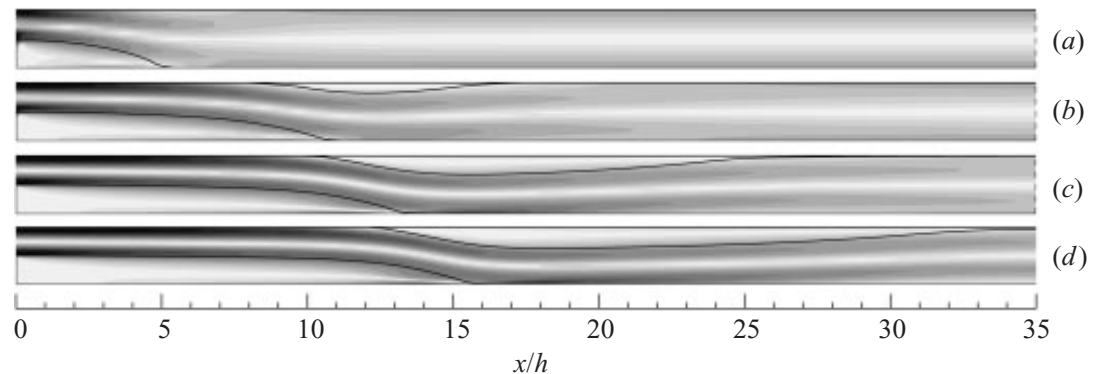

FiguRE 3. Visualization of the steady two-dimensional base flows at (a) $R e=150,(b) 450,(c)$ 750 and $(d)$ 1050. Each image shows the separation streamlines associated with the primary and secondary recirculation zones. Shaded regions correspond to vorticity magnitude in the range $0 \leqslant\left|\omega h / U_{\infty}\right| \leqslant 2$. Note the appearance of a separation bubble on the upper wall at $R e \simeq 300$ (between the $(a)$ and $(b)$ ). The dashed lines on the right indicate the outflow boundary of the computational domain. For $(d) L_{o}=45 h$ and the outflow boundary is outside the range shown.

\subsection{Convergence tests}

Flow past a backward-facing step is a deceptively difficult problem to fully resolve, especially at large Reynolds number. The combined effects of the geometric singularity at the step corner and the convective instability of the core flow can produce and then amplify numerical errors that mimic an intrinsic temporal flow instability. Gresho et al. (1993) illustrate the minimum resolution requirements at $R e=600$ for a variety of methods. In this section we outline our own convergence tests for both the base flow and stability calculations to establish that the numerical results we present are well-resolved.

\subsubsection{Base flow}

There are two central issues in checking convergence of the base flow calculations: external dimensions of the grid $\left(L_{i}\right.$ and $\left.L_{o}\right)$ and the degree of internal refinement. External dimensions can be determined from a simple parameter study, but the unstructured nature of the discretization makes the internal refinement study more difficult to organize. Below we highlight only representative tests near the threedimensional critical Reynolds number, although we did perform a large number of calculations to test both domain size and resolution over the full parameter range.

Figure 3 shows an overview of our base flow calculations in terms of steady-state vorticity fields and separation streamlines at various Reynolds numbers, and provides a qualitative picture of how the base-flow structure evolves with increasing $R e$. At moderate Reynolds number the steady flow consists of a primary separation bubble extending from the step and a secondary separation bubble that first appears on the upper wall at $R e \simeq 300$. This secondary separation bubble was first reported by Armaly et al. (1983) and has been confirmed in numerous computational studies (e.g. Ghia et al. 1989; Gartling 1990; Kaiktsis et al. 1991; Williams \& Baker 1997).

The skin-friction coefficient, $C_{f}$, passes through zero exactly at the boundaries of the separation zones. This quantity provides a sensitive measure of the overall grid resolution. In the present case the skin-friction coefficient can be defined as

$$
C_{f} \equiv-\frac{2 v}{U_{\infty}^{2}} \frac{\partial u}{\partial y} n_{y}
$$

where $n_{y}$ accounts for the direction of the outward normal to the fluid: $n_{y}=1$ 

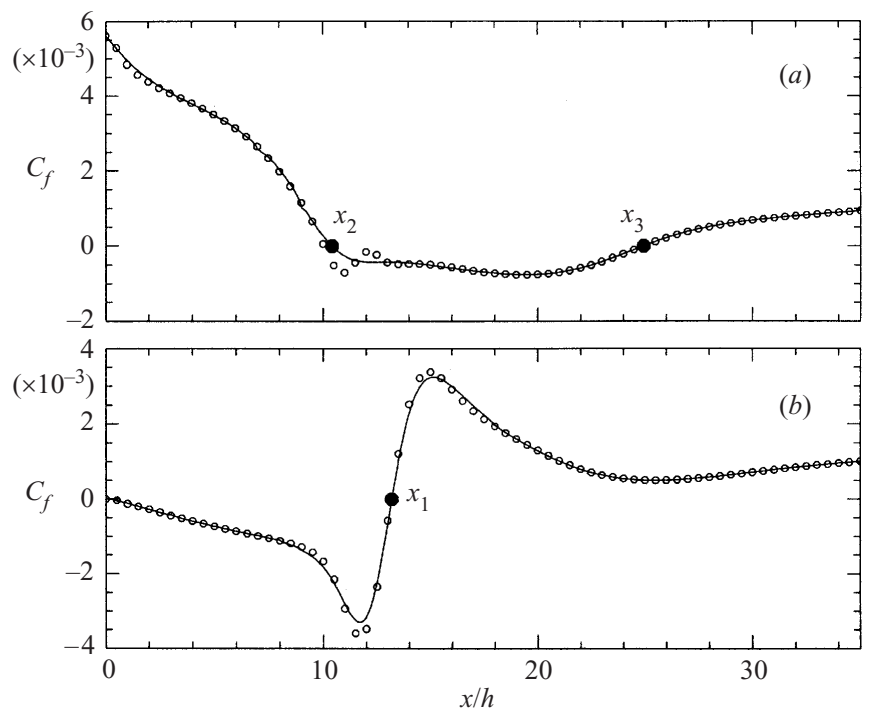

Figure 4. Computed skin-friction coefficients at $R e=750$ along $(a)$ the upper wall and $(b)$ the lower wall of the channel. Solid circles $(\bullet)$ mark the three downstream locations where $C_{f}\left(x_{j}\right)=0$. Open circles $(\bigcirc)$ indicate results from a coarse grid, $M_{3 a}$ with $N_{b}=7$, while the solid lines (-) indicate results from a fine grid, $M_{3 b}$ with $N_{b}=7$. Note the oscillations in the coarse-grid solution near $C_{f}\left(x_{2}\right)$; this is typical of a high-order method on an under-resolved grid.

on the upper wall and $n_{y}=-1$ on the lower wall. Although this quantity is only evaluated along the walls, its downstream distribution obviously depends on the dissipation of momentum in the core part of the flow. Figure 4 shows the computed distribution of $C_{f}$ for both a coarse and fine grid calculation at $R e=750$. The coarse and fine grids are based on the $M_{3 a}$ and $M_{3 b}$ meshes, respectively, shown in figure 2. Both calculations were run with a fixed polynomial order of $N_{b}=7$. Even though the spectral-element method does not impose continuity of derivatives at element boundaries, continuous velocity gradients are obtained for sufficiently high polynomial order. This is evidenced by the fact that our $C_{f}$ distributions appear continuous in the downstream direction. Oscillations in the solution along the upper wall suggest that the flow is 'under-resolved' on the coarse grid. These oscillations disappear upon grid refinement and the entire distribution converges to a smooth curve. Both solutions agree extremely well in the regions of the flow where $C_{f}$ varies slowly.

To further quantify the base flow calculations, we track the position of separationreattachment points in the domain as a function of Reynolds number, i.e. the set of locations along the walls where $C_{f}\left(x_{j}\right)=0$. Figure 4 indicates the location of these points for $R e=750$ based on the converged fine-grid solution. Since the extent of the primary and secondary separation zones varies with Reynolds number, the location of these points provides another good test of domain size and appropriate resolution. In particular it indicates the minimum external dimensions of the computational domain required to enclose the separation zones.

Figure 5 compares calculations on a variety of grids over the entire range of Reynolds number considered in our study. Our basic criterion for choosing $L_{o}$ is to locate it five or more step heights downstream of the reattachment point $x_{3}$. We have overlaid results for grid pairs $M_{k}$ and $M_{k+1}$ in figure 5, with at least one overlap 


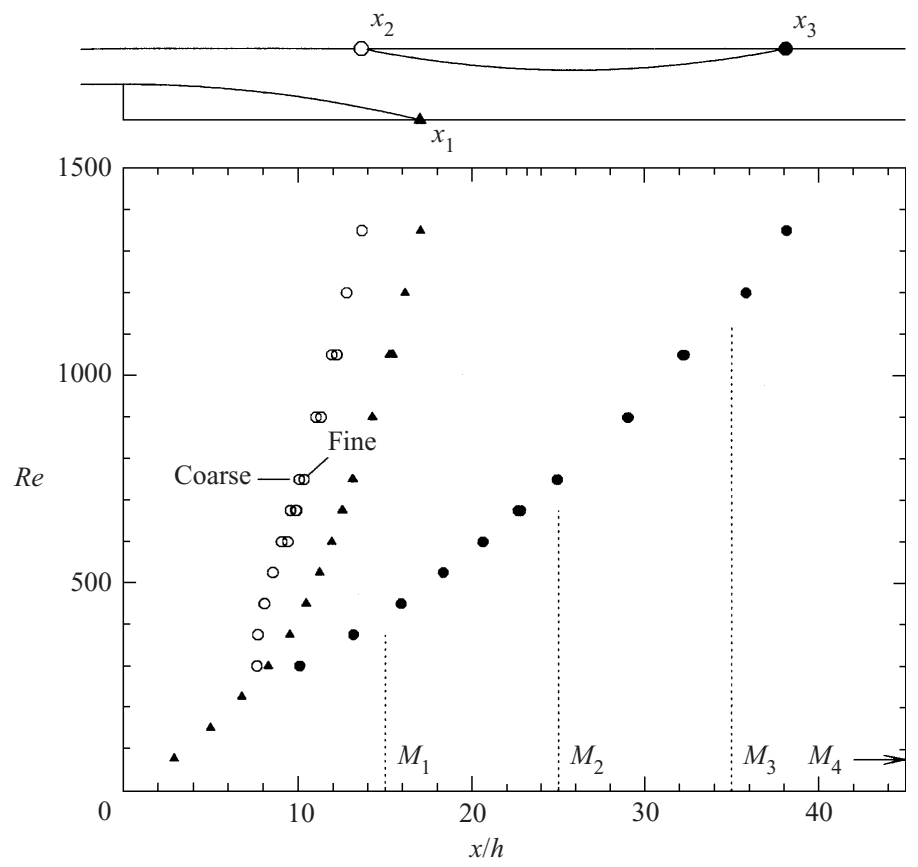

FIGURE 5. Separation-reattachment points in the backward-facing step flow as a function of Reynolds number. This figure compares results for several meshes with different geometries and internal levels of refinement. Dashed lines mark the location of the downstream boundary $\left(L_{o}\right)$ for each mesh, with the vertical extent indicating the valid range in $R e$ for each.

point for each consecutive grid pair. On the scale of the plot these overlaid points can barely be distinguished, thus showing that the computed reattachment points are insensitive to $L_{o}$ for the indicated Reynolds number range for each mesh. Consistent with the sensitivity of $C_{f}$ shown in figure 4 , the location of the separation point $x_{2}$ does show some sensitivity to the degree of internal refinement, with $x_{2}$ shifting just slightly downstream on the finer grid.

The results in figure 5 agree well with published computational data. Below $R e=300$ the computed primary separation length also agrees with the experimental data of Armaly et al. (1983), although those experiments were performed with and expansion ratio of 1.94 rather 2 . We also find that the secondary separation zone first appears at $R e \simeq 300$, as reported by Armaly et al. (1983). At larger Reynolds numbers the primary separation length does not agree with the experimentally measured values because the experimental flow is three-dimensional. This effect is well documented by Williams \& Baker (1997). However, our results do agree well with other two-dimensional computations in this regime. In particular, Gartling (1990) reports from highly resolved computations that at $R e=600$ these points occur at $x_{1}=12.2$, $x_{2}=9.7$, and $x_{3}=21.0$. The corresponding values from our fine-grid calculations are: $x_{1}=11.91, x_{2}=9.5$, and $x_{3}=20.6$, giving a discrepancy of only about $2 \%$. Discrepancies at this level may be attributed to the slightly different inflow boundary conditions used in each calculation.

Based on our convergence study we chose the following strategy to generate gridindependent base flows. First we performed a quick scan of Reynolds number using relatively coarse grids but with an appropriate $L_{o}$. Then we introduced internal 


$\begin{array}{ccccccc}N_{b} & N_{e} & \sigma_{1} & \sigma_{2} & \sigma_{3} & \omega_{3} & \sigma_{1}^{\prime} \\ 7 & 5 & 0.000129 & -0.012811 & -0.014109 & 0.031406 & 0.005919 \\ 7 & 7 & 0.000042 & -0.012898 & -0.014236 & 0.031633 & 0.004985 \\ 9 & 7 & 0.000033 & -0.012966 & -0.014234 & 0.031615 & 0.004927 \\ 9 & 9 & 0.000030 & -0.012972 & -0.014247 & 0.031615 & 0.004903\end{array}$

TABle 2. Dependence of eigenvalues on polynomial order. Parameters $N_{b}$ and $N_{e}$ indicate the independent polynomial order of the base flow and eigenmode. Three leading eigenvalues computed on the mesh $M_{3 b}$ at $R e=750, \beta=0.9$ are given; the first two eigenvalues $\sigma_{1}$ and $\sigma_{2}$ are real, the third is complex: $\sigma_{3} \pm \mathrm{i} \omega_{3}$. The leading eigenvalue $\sigma_{1}^{\prime}$ computed on the $M_{4}$ mesh at $R e=1050$, $\beta=1.5$ is given in the last column.

refinement and recomputed these flows to generate fine-grid solutions at a few Reynolds numbers bracketing points of interest, e.g. the primary bifurcation.

\subsubsection{Tests of stability calculations}

To quantify the precision of eigenpairs $(\mu, \tilde{u})$ produced by subspace iteration we compute the residual

$$
r=\left\|A_{U} \tilde{u}-\mu \tilde{u}\right\|,
$$

where $\|\cdot\|$ is the standard $L_{2}$ vector norm and the eigenmodes are scaled so that $\|\tilde{u}\|=1$. In most cases we continue iterating until $r<10^{-6}$ for one or more eigenmodes, although in calculations that generate several modes the residuals for the dominant ones are orders of magnitude smaller than this. For practical purposes these can be considered exact eigenvalues of the operator $A_{U}$ so that the true accuracy is determined by how well the numerical operator $A_{U}$ approximates the linear stability operator for the continuous problem.

Since accurate eigenvalue computations are predicated on having an accurate base flow $U$, the grid requirements (external dimensions and level of internal refinement) are dictated to a large degree by the base flow considerations discussed previously. However, we frequently use a lower polynomial order for the stability computations, particularly when scanning a large range of spanwise wavenumbers for a fixed base flow.

To demonstrate the accuracy of the eigenvalue computations, we present in table 2 results as a function of base flow and eigenmode polynomial order, $N_{b}$ and $N_{e}$. We give the leading eigenvalues at $R e=750, \beta=0.9$, values extremely close to the primary instability, and also at $R e=1050, \beta=1.5$, values near those giving the largest growth rate found in our study. In all cases the residual satisfies $r<10^{-8}$ for the leading eigenvalues $\sigma_{1}$ and $\sigma_{1}^{\prime}$, and satisfies $r<10^{-6}$ for the other eigenvalues. The eigenvalues have converged to an absolute accuracy of less than $10^{-4}$ at the highest resolution $\left(N_{b}=N_{e}=9\right)$. The relative error of $\sigma_{1}$ in table 2 is large (because $\sigma_{1} \approx 0$ ), but the absolute error is small and sufficient to determine the primary instability to high precision.

As an additional check for systematic errors in our computations, we computed the leading eigenvalue at $\operatorname{Re}=600$ for $\beta=0$, i.e. the two-dimensional stability problem. With $N_{b}=N_{e}=9$ using mesh $M_{3 b}$ we find $\sigma=-0.006288$. Gresho et al. (1993) obtained a leading eigenvalue $\sigma=-0.006277$ at this Reynolds number using a different formulation and different inflow and outflow lengths for the 
backward-facing step geometry. $\dagger$ Such good agreement with an independent calculation - a relative difference less than $0.2 \%$-gives us further confidence in our results.

Finally, as a test of the effect of outflow length on the stability computations we have computed the leading eigenvalue at $R e=1050$ for $\beta=0$ and $N_{b}=N_{e}=9$ using two outflow lengths. For the $M_{4}$ mesh (outflow $L_{0}=45$ ) we obtain $\sigma=-0.002896$. For a similar mesh with outflow $L_{0}=55$ we obtain $\sigma=-0.002904$, thus verifying that, just as for the base flow, $L_{0}=45$ gives a well-resolved result at this Reynolds number.

Our stability computations follow a similar protocol to that used for obtaining converged base flows. We initially compute eigenvalue branches using a moderate resolution. Typically we use the $M_{3 b}$ mesh with polynomial orders $N_{b}=7$ and $N_{e}=7$. Once the approximate location of a bifurcation point is known, we repeat the calculations in that vicinity at higher resolution, up to polynomial order $N_{b}=N_{e}=9$.

\section{Results}

\subsection{Parameter dependence}

We begin by summarizing our findings for the dependence of eigenvalues on Reynolds number and spanwise wavenumber $\beta$. Figure 6 shows the real part of the leading eigenvalues (those with largest real part) as a function of $\beta$ for three values of Reynolds number encompassing the primary instability. The eigenvalue curves are symmetric with respect to a change in sign of $\beta$ and only portions with $\beta \geqslant 0$ are plotted. Figure 7 shows the leading part of the spectrum at $R e=750, \beta=0.9$ (parameter values near the primary instability), and serves to illustrate where the eigenvalues plotted in figure 6 lie in the complex plane.

The three eigenvalue plots in figure 6 have much the same general character. There are two local maxima in the leading branch: one at $\beta \approx 0.15$ and the other at $\beta \approx 0.9$. Between these local maxima the two real eigenvalues join to form a complex-conjugate pair over a small range in $\beta$. For $R e=750$ and $R e=1050$ the leading eigenvalues become complex again at larger spanwise wavenumbers. There is also a separate branch of complex eigenvalues (also seen in figure 7) with real part comparable to that of the purely real eigenvalues. These complex eigenvalues do not become positive in the range of Reynolds number studied and thus play no active role in the instability of the flow. We have investigated wavenumbers larger than those shown in figure 6 and find no evidence of other eigenvalues that would give rise to instabilities in this Reynolds number range. Note that we have not plotted in figure 6 eigenvalues associated with damped downstream channel modes seen in figure 7. They are not relevant to linear instabilities at these Reynolds numbers.

From figure 6 it can be seen that the primary linear instability for the backwardfacing step occurs just below $R e=750$. The instability is three-dimensional with a spanwise wavenumber of $\beta \approx 0.9$. To determine the bifurcation point precisely we have computed the leading eigenvalues at $R e=750$ and $R e=735$ for several values of $\beta$ near 0.9 . From cubic polynomial fits to the eigenvalue data we find the local maximum in the eigenvalue branches at these two Reynolds numbers. We then

$\dagger$ The value quoted is the mesh $D$ value from Gresho et al. converted to our units. Their result is reported as a positive number in inverse diffusive time units, whereas we report eigenvalues with the opposite choice of sign in inverse advective time units. To convert to our non-dimensionalization their reported value $\sigma=15.06489$ must be made negative and multiplied by $v / 4 h U_{\infty}=1 / 2400$. 

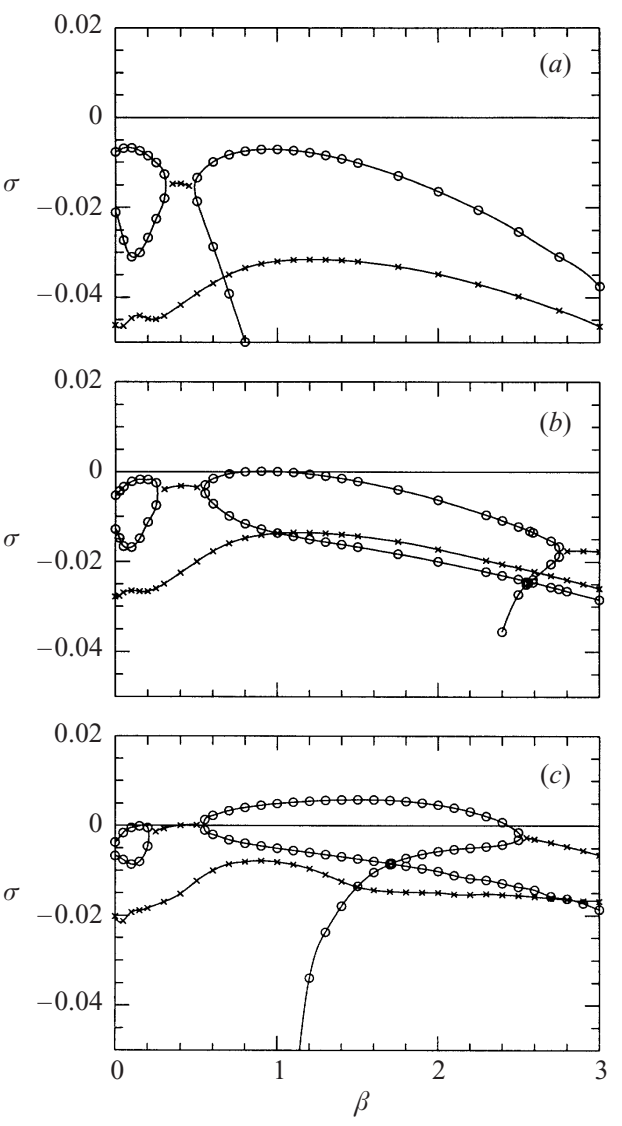

FiguRE 6. Leading eigenvalues at $(a) \operatorname{Re}=450,(b) \operatorname{Re}=750$ and $(c) \operatorname{Re}=1050$ as function of spanwise wavenumber. The real part $\sigma$ of eigenvalues $\sigma+\mathrm{i} \omega$ is plotted. Circles denote real eigenvalues $(\omega=0)$ and crosses denote complex eigenvalues $(\omega \neq 0)$. These results have been obtained on the $M_{3 b}$ mesh with polynomial orders $N_{b}=7, N_{e}=7$.

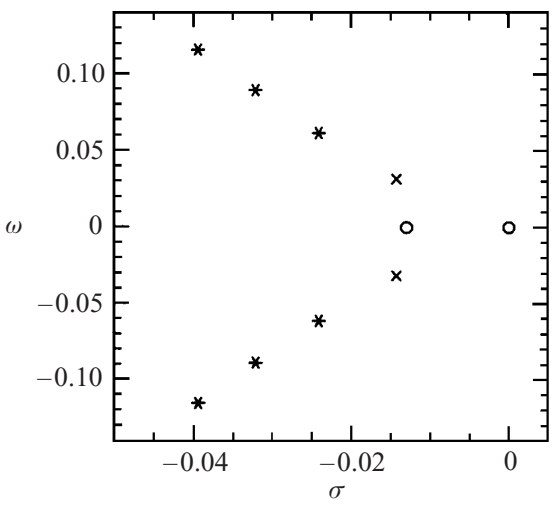

FIGURE 7. Stability spectrum at $R e=750$ for $\beta=0.9$ : $\bigcirc$, real eigenvalues; $\times$, complex eigenvalues associated with the step; *, complex eigenvalues associated with modes of the downstream channel (see $\S 4.2$ ). These latter eigenvalues depend on the outflow length $L_{o}$ used in the computation. These results have been obtained on the $M_{3 b}$ mesh with polynomial orders $N_{b}=N_{e}=7$. 


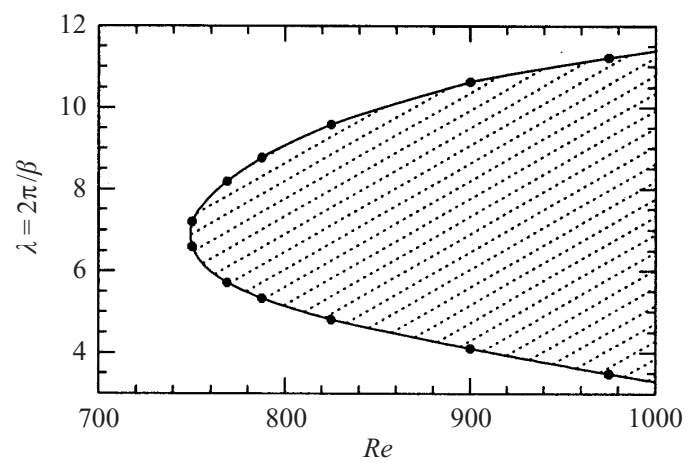

FIGURE 8. Neutral stability curve for backward-facing step flow. Everywhere in the shaded region the flow is linearly unstable to three-dimensional perturbations over a finite band of wavelengths. The points have been obtained on the $M_{3 b}$ mesh with polynomial orders $N_{b}=N_{e}=7$, and the curve is a fit to these data.

linearly interpolate between the maxima to find the Reynolds number and spanwise wavenumber at which the maximum crosses zero. We have done this for outflow lengths of $L_{o}=35$ and $L_{o}=45$ and several polynomial orders up to $N_{b}=N_{e}=9$. From these data we find the critical values for the primary instability to be $R e_{c}=748$ and $\beta_{c}=0.91$, to within an uncertainty of $1 \%$. The critical spanwise wavelength is $2 \pi / \beta_{c}=6.9$ step heights.

The critical Reynolds number that we find for the flow without lateral sidewalls is considerably larger than the Reynolds number at which Armaly et al. (1983) first report observing three-dimensional motions. In our units, Armaly et al. (1983) observed significant three-dimensionality at $R e=300$. Kaiktsis et al. (1991) report stable three-dimensional flows starting at Reynolds numbers of about 525 in spanwiseperiodic simulations, and Williams \& Baker (1997) find, in simulations with lateral sidewalls, some degree of three-dimensionality even below $R e=300$. There are several reasons for these discrepancies which we discuss fully in section $\S 5$.

In figure 8 we plot the neutral stability curve for the backward-facing step flow up to Reynolds number 1000 . Everywhere to the right of the curve the flow has at least one positive eigenvalue and is therefore linearly unstable. Points along the curve were obtained by locating the zero crossings of eigenvalue branches as a function of $\beta$ for several fixed Reynolds numbers between 750 and 1000. The neutral stability curve becomes more complicated just above $R e=1000$ because a complex-eigenvalue portion of the leading branch crosses the imaginary axis (this can be seen in figure 6(c) for $R e=1050, \beta \sim 0.5$ ). However, this is far above the primary instability.

\subsection{Eigenmodes}

We turn now to the structure of the linear mode that destabilizes the basic twodimensional flow. Figures 9 and 10 show the leading eigenmode at $R e=750, \beta=0.9$. This is essentially the critical eigenmode. The spanwise-velocity contours of figure 10 clearly show that the bifurcating mode is localized to the primary recirculation region downstream of the step. The instability involves neither the bulk flow nor the secondary separation bubble to any significant degree. While the exact size and shape of the primary recirculation zone depends on the global flow properties, the three-dimensional instability is driven by this local part of the flow field.

The cross-channel velocity ( $y$-velocity) in the critical eigenmode is quite small everywhere (figure 9); the spanwise velocity component is particularly strong just 


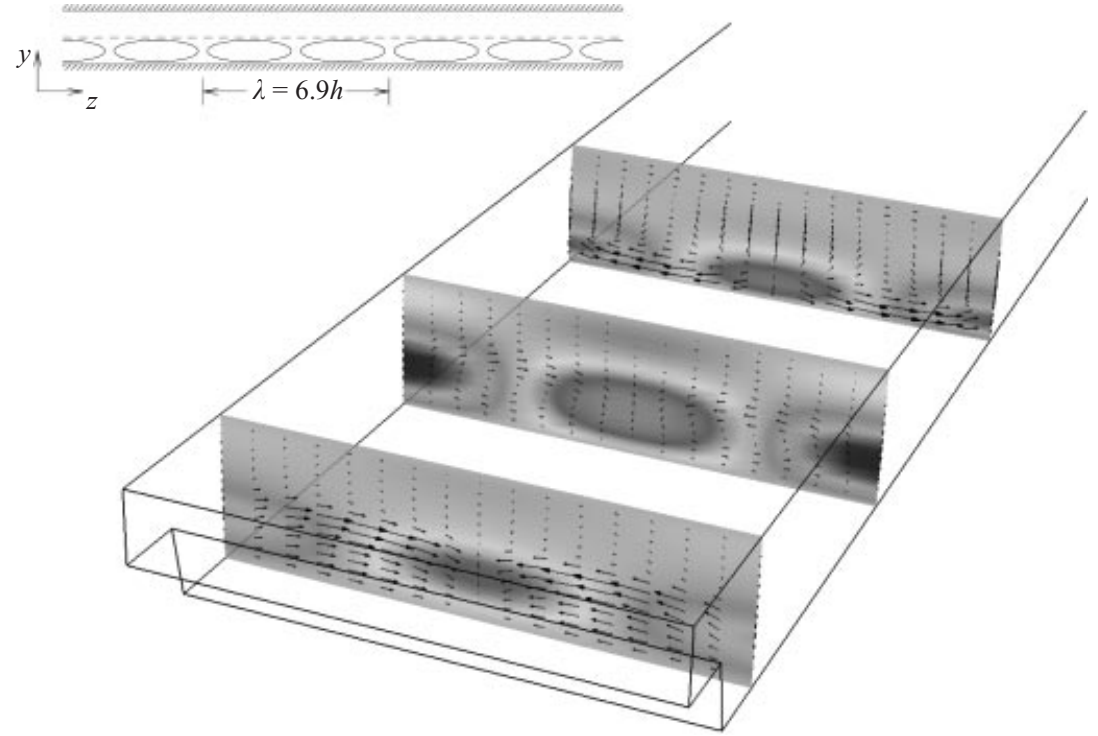

FIGURE 9. Three-dimensional flow structure of the critical eigenmode at $R e=750$ and $\beta=0.9$. Contours indicate the strength of the streamwise velocity component and vectors show the $(v, w)$ flow pattern in each cross-sectional plane: $x=1.2,6.2$ and 12.2 .

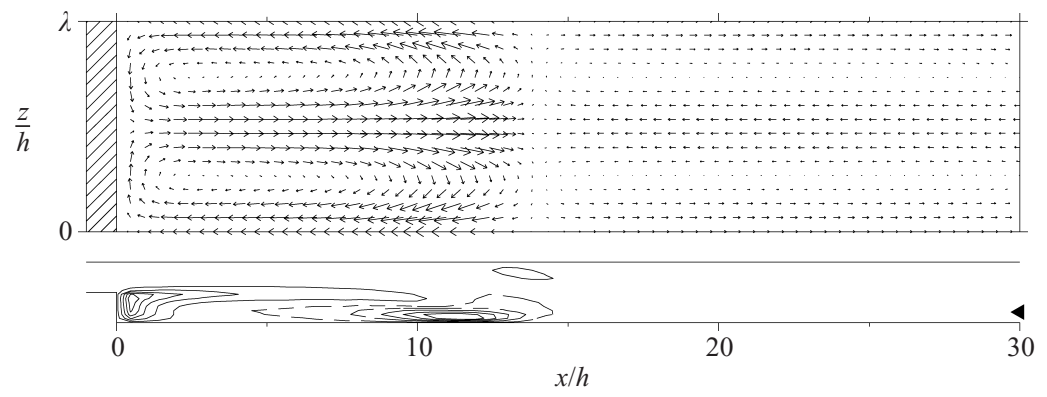

FIGURE 10. Sections of the critical three-dimensional eigenmode. Upper plot show $(u, w)$ velocity vectors in the plane $y=-0.65$ (indicated by a triangle at the right of the lower plot). The lower plot contains $w$ velocity contours (in the plane $z=\lambda / 4$ ) with solid and dashed contours indicating the sign of $w$.

behind the step edge and at the downstream reattachment point $\left(x_{1} \simeq 13.2\right)$. The secondary flow generated by the instability can best be described as a flat roll lying within the primary recirculation zone. This flow is qualitatively similar to the transient secondary flow sketched by Denham \& Patrick (1974) following perturbations to the two-dimensional flow at Reynolds number 344. However, the spanwise wavelength of $6.9 \mathrm{~h}$ is considerably smaller than that reported by Denham \& Patrick (1974). We make further comparison with experiment in section $\S 5$.

Figure 11 shows the modes associated with the next three largest eigenvalues in the spectrum at $R e=750$ and $\beta=0.9$ (see table 2 and figure 7). The next real eigenmode (corresponding to $\sigma_{2} \simeq-0.013$ ) plotted in figure 11(a) is localized to the secondary separation region on the upper wall. The separation and reattachment points on the upper wall are located at $x_{2} \simeq 10$ and $x_{3} \simeq 25$, respectively. This secondary mode is also a flat roll. Thus, the two largest real eigenvalues correspond to similar physical 

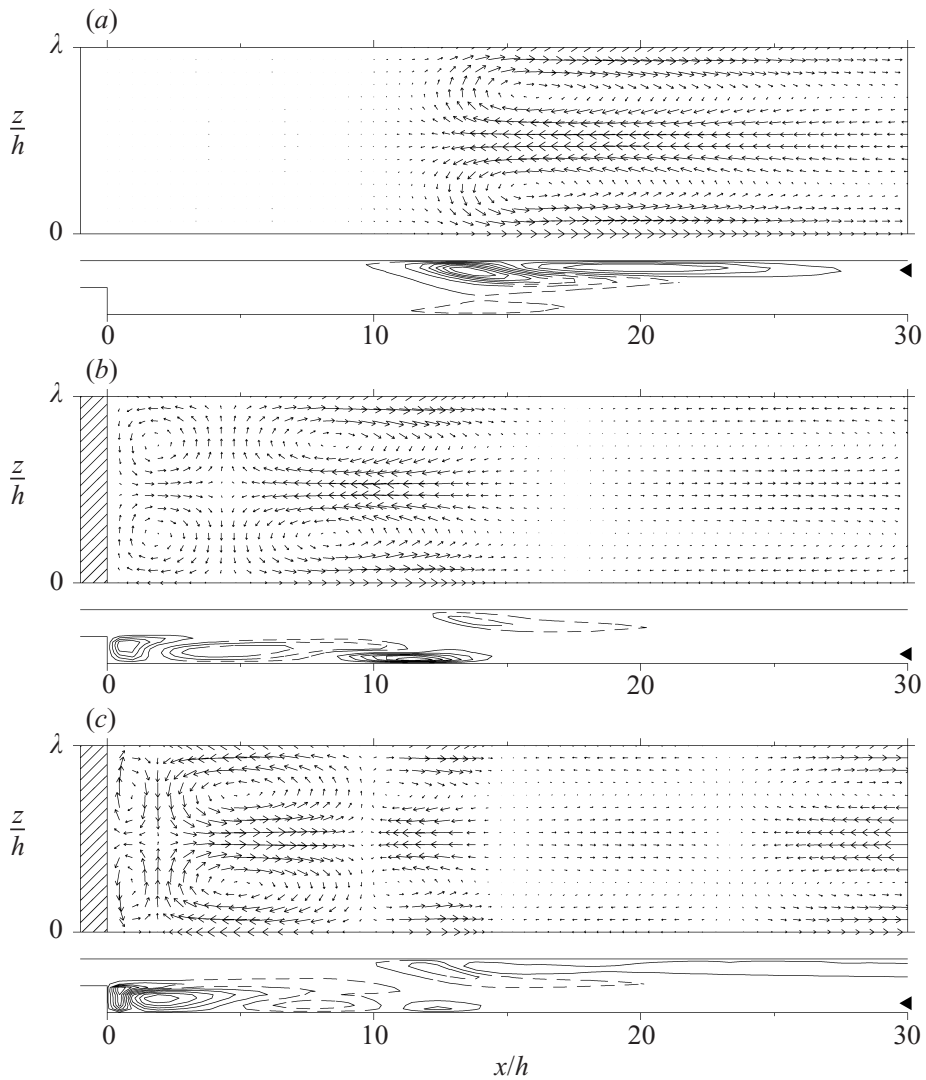

FiguRE 11. Structure of the next three eigenmodes at $R e=750$ and $\beta=0.9:(a)$ real eigenmode corresponding to eigenvalue $\sigma_{2} ;(b)$ and $(c)$ real and imaginary parts of the complex eigenmode corresponding to eigenvalues $\sigma_{3} \pm \mathrm{i} \omega_{3}$. In each case the upper plot show $(u, w)$ velocity vectors in the $y$-plane indicated at the right of the lower plot. The lower plot contains $w$ velocity contours in the plane $z=\lambda / 4$.

modes but are associated with quite different regions of the flow. The first complex mode is displayed in figure $11(b, c)$ in terms of its real and imaginary parts. The dynamics associated with this mode is a periodic oscillation between the two states. While it is principally associated with the primary recirculation region, this mode does extend into the upper recirculation region as well. The flow has a more complicated spatial structure with several 'rolls' within the primary recirculation region: the sign of the spanwise velocity component changes two (figure 11b) and three (figure 11c) times.

Finally, for completeness we show in figure 12 one of the channel modes whose eigenvalues are plotted in figure 7 (the one with right-most eigenvalue). As is evident from the spectrum, numerous similar modes are found in our computations. However, these modes are irrelevant to the linear instability of the step flow and we do not consider them further.

\subsection{Two-dimensional stability}

The results of $\S 4.1$ show that up to a Reynolds number of 1050 the two-dimensional flow over the backward-facing step is linearly stable with respect to two-dimensional perturbations, that is, all eigenvalues at $\beta=0$ have negative real part. The question 


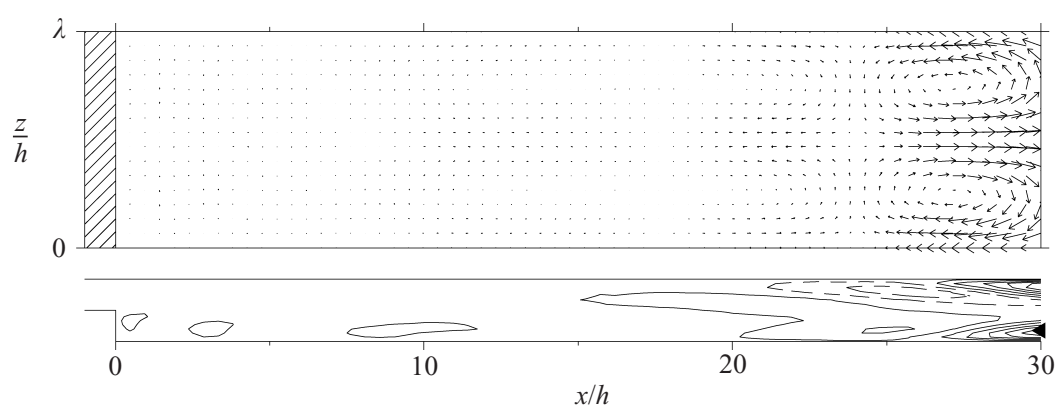

FIGURE 12. Structure of the channel eigenmode corresponding to the channel eigenvalue with largest real part. Only the real part of the complex eigenmode is plotted.

arises of when the flow becomes linearly unstable two-dimensionally. This has been a point of some controversy in previous computational studies of the two-dimensional flow (see Gresho et al. 1993). While this is not the focus of our study and our numerical methods have not been designed to study large Reynolds numbers for this flow, we have nevertheless continued the two-dimensional stability computations to $R e=1500$, twice the critical value for the onset of three-dimensional instability. The flow remains linearly stable to two-dimensional perturbations and moreover shows no evidence of any nearby two-dimensional bifurcation. Because accurate computations become very demanding at large Reynolds numbers and because there is no evidence that an instability is at hand, we cannot report a threshold for two-dimensional instability but only a lower bound for this threshold.

In figure 13 we plot, as a function of Reynolds number, the two leading eigenvalues from strictly two-dimensional stability computations, i.e. modes of the form

$$
\tilde{\boldsymbol{u}}(x, y)=(\hat{u}(x, y), \hat{v}(x, y), 0), \quad \tilde{p}(x, y)=\hat{p}(x, y) .
$$

Eigenvalues for all Reynolds numbers greater than 1050 have been computed with outflow length $L_{o}=55$ and with polynomial order $N=9$. For lower Reynolds numbers, shorter domains have been used consistent with convergence studies in $\S 3.3$. Recall (§3.1) that the two-dimensional base flows above $R e=800$ are computed by time integration to a steady state. While this computational method is slow, it has the advantage here of confirming that all of the steady two-dimensional flows we have computed are globally stable with respect to two-dimensional perturbations.

The two eigenvalue branches approach one another at $R e \approx 1250$. Generically, as the point of intersection is approached, the eigenvalues will either coalesce in a complex conjugate pair or will instead remain real and undergo avoided crossing. We find that the leading eigenvalues plotted in figure 13 remain real.

The eigenvalues have been plotted on a logarithmic scale to show the approximate exponential dependence of the eigenvalues on Reynolds number (taking into account the exchange of identity through the avoided crossing). The plot shows that if the trend continues, these eigenvalues will reach zero only at infinite Reynolds number. We do not argue that this is what actually happens, but we can state that up to the Reynolds number that we have been able to attain in our calculations, there is no two-dimensional instability of the flow and moreover that there is no evidence of any two-dimensional bifurcation close to these Reynolds numbers. To ascertain the primary two-dimensional bifurcation for this flow, other numerical methods will need to be employed. 


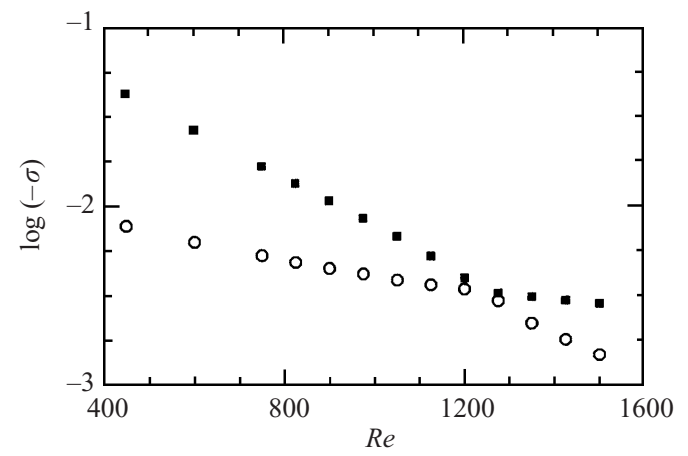

FIGURE 13. Leading two-dimensional eigenvalues. The two branches (denoted by different symbols) remain real as they approach and recede from one another through the avoided crossing at $R e \approx 1250$.

\section{Discussion}

A detailed comparison between our linear stability calculations and either previous experimental work or direct numerical simulations is complicated by several factors. Among these are the existence of a strong convective instability within the core flow, the variety of geometries used in previous studies (expansion ratio, aspect ratio, sidewalls, no sidewalls), different upstream flow conditions, and so forth. Of these effects, the convective instability within the core flow is the most problematic as it renders the system particularly sensitive to upstream conditions, including free-stream noise and external perturbations. The flow may amplify selective components of these perturbations to produce apparent three-dimensionality and unsteadiness well before the onset of any absolute instability. The combined effects of these discrepancies mean that there is no single experiment or computation with which we can make a detailed comparison. In the following we compare our results with data available in the existing literature and try to indicate what further work might help clarify the nature of the absolute instability.

\subsection{Comparison with previous work}

We begin by comparing our results with the experimental work of Armaly et al. (1983) and the related computations by Williams \& Baker (1997) in a system with a nominal expansion ratio of 2 and aspect ratio $L_{z} / h=37$. In both the experiments and computations the span of the channel terminated at a solid wall on both sides. These investigators found that below $R e=300$ the flow is essentially spanwise invariant while above $R e=300$ there is evidence of three-dimensionality in the flow. The value $R e=300$ does not necessarily indicate a critical point. Williams \& Baker (1997) note that some deviation from two-dimensionality exists near the sidewalls of the channel below $R e=300$, and neither study attempted to pinpoint the value of $R e$ at which significant three-dimensionality along the span first appeared in the system. Even the characterization of 'significant' is arbitrary.

The first question to address is whether these results indicate a fundamental threedimensional instability at a lower Reynolds number, as these observations are well below the critical value $R e_{c}=748$. The source of the discrepancy is almost certainly the effect of sidewalls in the experiments and simulations. Williams \& Baker (1997) provide evidence that, in the presence of sidewalls, three-dimensionality in these flows (also observed in the experiments of Armaly et al.), is caused by a sidewall jet. In fact, 
both studies indicate that three-dimensionality is limited to the region within a few step heights of the sidewalls while the bulk flow remains largely spanwise-invariant at these low Reynolds numbers. This type of extrinsic effect is produced by the threedimensional geometry of the laboratory setup, and it will surely depend on the aspect ratio of the system. It does not represent a fundamental instability of the nominally two-dimensional separated flow.

There is further computational evidence that the sidewalls play an important role. In the three-dimensional simulations of Kaiktsis et al. (1991) using spanwise-periodic boundary conditions, sustained three-dimensionality was first found at a much higher Reynolds number, $R e \simeq 525$, than was found in the numerical studies with sidewalls by Williams \& Baker (1997). Interestingly, Kaiktsis et al. (1991) used a spanwise-periodic domain of length $2 \pi$, almost exactly the critical wavelength $\lambda_{c}=6.9 h$ determined by our analysis. However, even without sidewall effects the onset of three-dimensionality in the work of Kaiktsis et al. (1991) is still below $R e_{c}$. There are two possible explanations for this.

The first is that the states observed by Kaiktsis et al. (1991) follow from amplification of numerical noise due to poor resolution in the form of a convective instability. In later work, Kaiktsis et al. (1996) show this to be the source of the sustained two-dimensional dynamics observed by Kaiktsis et al. (1991) at $R e=600$. Our computations are free from any such effects in that we determine only global absolute instability thresholds. However, we have performed preliminary nonlinear calculations of the three-dimensional flow, i.e. direct numerical simulations, that do confirm the existence of a strongly three-dimensional convective instability. These dynamics are poorly understood.

The other possible explanation for sustained three-dimensionality below $R e_{c}$ is that the instability is subcritical. From our linear computations we cannot know whether or not stable, nonlinear, three-dimensional states exist below the linear stability threshold. Whether or not this is the case, the simulation results of Kaiktsis et al. (1991, figures 11 and 24) indicate strong three-dimensionality primarily in the region downstream of the separation zone, and bear little qualitative resemblance to the critical eigenmode. Therefore it seems unlikely that their results provide any evidence of a subcritical bifurcation due to the absolute instability of the flow. This is an interesting point, but further computational work is required to assess whether or not the three-dimensional bifurcation is subcritical.

In addition to the question of critical Reynolds number, there is the character of the secondary flow following the bifurcation. Denham \& Patrick (1974) conducted experiments on laminar flow in a channel with a $3: 1$ expansion ratio for Reynolds numbers up to 344. It has been noted (Ghia et al. 1989; Williams \& Baker 1997) that the relatively short inlet channel in these experiments resulted in a slightly shortened primary recirculation zone. Despite this, and the fact that the expansion ratio used by Denham \& Patrick (1974) is larger than ours, we can still make a useful comparison because they report a transient three-dimensional flow qualitatively similar to that of the critical eigenmode. Experimentally, the flow at $R e=344$ was perturbed by tapping the channel. The observed transient flow consisted of horizontal eddies within the recirculation zone. Moreover, this transient flow was found to be the forerunner of waves and then eddies that appear as the Reynolds number is further increased, though none of this behaviour was described in detail. Denham \& Patrick (1974) sketch only two pairs of secondary eddies over the channel span $L_{z} \simeq 20 h$, from which we conclude that the size of these eddies is larger that the critical wavelength $\lambda_{c}=6$.9. It is not clear, however, how precise the sketch is. Recall also that there are 
two peaks in the eigenvalue spectra in figure 6, and at lower Reynolds numbers the small- $\beta$ peak actually corresponds to a larger eigenvalue, i.e. a slower decay rate. It is therefore possible that Denham \& Patrick (1974) have observed evidence of these three-dimensional modes. Other researchers have also reported dynamics within the primary recirculation region, but it is not clear how to connect these flows to the eigenmode.

\subsection{Instability mechanisms}

Our stability calculations are unambiguous with regard to the critical Reynolds number, wavelength, and flow structure associated with the absolute instability. Here we address the question of why the flow amplifies this particular type of perturbation.

One obvious a priori candidate is the Kelvin-Helmholtz instability of the shear layer emanating from the step edge. Although the Kelvin-Helmholtz instability is important at higher Reynolds number and as a source of amplification in the context of convective instability, it plays no role here because the three-dimensional instability is absolute. Furthermore, because of the relative thickness of the shear layer at these Reynolds numbers, and the stabilizing effect of the walls, it is difficult to excite any shear layer response in this parameter range.

Armaly et al. find that three-dimensionality first appears close to the Reynolds number at which the upper separation bubble forms. Ghia et al. (1989) postulate that once the secondary separation bubble forms, the main flow is subjected to a destabilizing concave curvature resulting in a three-dimensional, Taylor-Görtlertype instability. This is an interesting speculation. However, from our linear stability computations we can also rule this out. We find not only that the two-dimensional flow remains linearly stable long after the formation of the upper separation bubble, but also that when instability does set in, it is not of the form of streamwise vortices within the main flow as would be expected by this mechanism.

We argue that the essential mechanism is still centrifugal in nature but is associated with the closed streamlines in the primary recirculation zone near the solid boundaries. The basic inviscid condition for a centrifugal instability is Rayleigh's criterion (e.g. Drazin \& Reid 1981) and its generalization by Bayly (1988). Physically, in a flow with closed streamlines one expects instability to arise if there is an outward decrease in the magnitude of angular momentum. To investigate this, let $\eta \equiv-\partial|\boldsymbol{r} \times \boldsymbol{u}|^{2} / \partial r$, where $\boldsymbol{r}=\left(x-x_{c}, y-y_{c}\right)$ with $\left(x_{c}, y_{c}\right)$ the centre about which the angular momentum is defined. We take this to be the point where the velocity vanishes, but in fact the results shown below depend only weakly on the choice of $\left(x_{c}, y_{c}\right)$ for any reasonable choice. The flow is (inviscidly) centrifugally unstable where $\eta>0$.

Figure 14(a) shows the regions inside the primary recirculation zone where $\eta$ is significantly positive for the two-dimensional flow at the critical Reynolds number. The regions where the magnitude of the angular momentum decreases significantly radially outwards are just behind the step face and just upstream of the re-attachment point. (All along the bottom wall and the step face $\eta$ is small and positive. For clarity we do not show regions where $\eta$ is less than $0.5 \%$ of the maximum value of $\eta=0.13$.) The regions shown in figure $14(a)$ are those for which the inviscid Rayleigh criterion predicts three-dimensional instability. Note that these are indeed the regions in which the magnitude of spanwise perturbation velocity is largest. See figure 14(b).

Intuitively the regions where the perturbation field has a significant three-dimensional component are where one would expect centrifugal effects to be most important. The streamlines have large curvature and the presence of walls dictates an outwardly decreasing momentum. The centrifugal instability pushes fluid away from the centre 


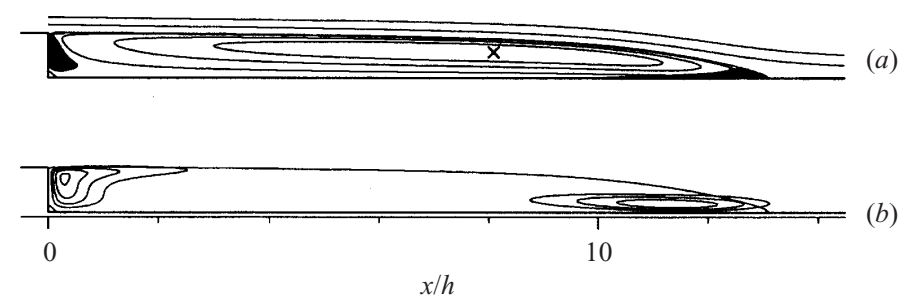

FIGURE 14. (a) Regions (black) where the magnitude of the angular momentum decreases away from the centre (marked with a cross) of the primary recirculation zone for the two-dimensional flow at Reynolds number 750. Specifically, the regions are shown where $\eta$ is greater than $0.5 \%$ of its maximum value. Also shown are representative streamlines. (b) Contours of the magnitude of the spanwise velocity component $w$ in the critical eigenmode. The separating streamline of the base flow is also shown.

of rotation, and the presence of the walls forces it to flow along the span to form the flat roll structure observed in the eigenmode. The spanwise length scale is not directly related to the step height but instead depends on the length of the separation bubble within which these three-dimensional eddies are generated.

The centrifugal mechanism is fundamentally three-dimensional in nature. This explains our finding that the backward-facing step flow does not become unstable two-dimensionally even at Reynolds numbers of twice the critical value for the onset of the three-dimensional instability. Some other mechanism would need to come into play at larger Reynolds numbers in order for this flow to become two-dimensionally unstable. For example, the upper separation bubble may become globally unstable (Hammond \& Redekopp 1998; Alam \& Sandham 2000). The flow will certainly become linearly unstable at $R e=2 \times 5772$ (e.g. Bayly et al. 1988) when flow in the downstream channel becomes unstable (the factor of 2 accounts for the difference between the Reynolds number used in this paper and the Reynolds number which applies to the downstream channel).

\section{Conclusion}

We have shown that the primary bifurcation of the steady, two-dimensional flow over a backward-facing step with a 2:1 expansion is a steady, three-dimensional instability. We have computed the critical Reynolds number and spanwise wavelength of the instability to high precision and find $R e_{c}=748$ and $\lambda_{c}=6.9$ in non-dimensional units based on the step height and the centreline velocity of the inflow. We have further determined the band of unstable wavenumbers for Reynolds numbers up to 1000 . These data will be particularly useful in future numerical work as they allow the precise selection of appropriate spanwise domain lengths.

We have found that the critical eigenmode consists of a flat roll localized to the primary recirculation region located behind the step edge. Thus, at the linear level, the instability does not arise in either the secondary recirculation zone on the wall opposite the step or the core flow between the primary and secondary recirculation zones. From this we have been able to rule out a Taylor-Görtler-type instability of the main flow as the source of three-dimensionality in experiments, but have argued that centrifugal instability is responsible for generating secondary flow with the separation zone.

Finally, we have examined the two-dimensional stability problem, and while we have not found a two-dimensional bifurcation from the steady flow, we have established 
a lower limit for such a bifurcation of $R e=1500$, considerably above the critical Reynolds number for three-dimensional instability. This establishes the fundamental role of three-dimensionality for separated flows similar to this one.

Following on from this work and the work of Kaiktsis et al. (1996), future studies should be conducted to examine three-dimensional convective instabilities of the backward-facing step. In the same way it would also be important to extend this work to nonlinear stability computations and determine whether the bifurcations are supercritical or subcritical.

We thank G. Brown and P. Marcus for many useful discussions on the topic of centrifugal instability. M. G. M. G. acknowledges support from the EPSRC, UK and the FCT, Portugal, and the hospitality of the IMA, University of Minnesota, where part of this research was carried out. R. H.H. acknowledges support from the NSF.

\section{REFERENCES}

AdAms, E. W. \& Johnston, J. P. 1988 Effects of the separating shear-layer on the reattachment flow structure part 2: reattachment length and wall shear-stress. Exps. Fluids 6, 493-499.

Akselvoll, K. \& Moin, P. 1993 Large eddy simulation of a backward-facing step flow. In Engineering Turbulence Modeling and Experiments 2 (ed. W. Rodi \& F. Martelli), pp. 303-313. Elsevier.

Alam, M. \& SANDham, N. D. 2000 Direct numerical simulation of 'short' laminar separation bubbles with turbulent reattachment. J. Fluid Mech. 410, 1-28.

Armaly, B. F., Durst, F., Pereira, J. C. F. \& Schönung, B. 1983 Experimental and theoretical investigation of backward-facing step flow. J. Fluid Mech. 127, 473-496.

Avva, R. K. 1988 Computation of the turbulent flow over a backward-facing step using the zonal modeling approach. PhD thesis, Stanford University.

Barkley, D. \& Henderson, R. D. 1996 Three-dimensional Floquet stability analysis of the wake of a circular cylinder. J. Fluid Mech. 322, 215-241.

Barkley, D. \& Tuckerman, L. S. 1999 Stability analysis of perturbed plane Couette flow. Phys. Fluids A 11, 1187-1195.

BAYLY, B. J. 1988 Three-dimensional centrifugal-type instabilities in inviscid two-dimensional flows. Phys. Fluids 31, 56-64.

Bayly, B. J., Orszag, S. A. \& Herbert, T. 1988 Instability mechanisms in shear-flow transition. Annu. Rev. Fluid Mech. 20, 359-391.

Bernardi, C., Maday, Y. \& Patera, A. T. 1992 A new nonconforming approach to domain decomposition: the mortar element method. In Nonlinear Partial Differential Equations and their Application (ed. H. Brezis \& J. L. Lyons). Pitman and Wiley.

Butler, K. M. \& FARrell, B. F. 1993 Optimal perturbations and streak spacing in wall-bounded turbulent shear-flow. Phys. Fluids A 5, 774-777.

Denham, M. K. \& Patrick, M. A. 1974 Laminar flow over a downstream-facing step in a twodimensional flow channel. Trans. Inst. Chem. Engrs 52, 361-367.

Drazin, P. G. \& ReID, W. H. 1981 Hydrodynamic Stability. Cambridge University Press.

Edwards, W. S., Tuckerman, L. S., Friesner, R. A. \& Sorensen, D. C. 1994 Krylov methods for the incompressible Navier-Stokes equations. J. Comput. Phys. 110, 82-102.

Fortin, A., Jardak, M., Gervais, J. J. \& Pierre, R. 1997 Localization of Hopf bifurcations in fluid flow problems. Intl J. Numer. Meth. Fluids 24, 1185-1210.

GartLING, D. K. 1990 A test problem for outflow boundary-conditions - flow over a backward-facing step. Intl J. Numer. Meth. Fluids 11, 953-967.

Ghia, K. N., Osswald, G. A. \& Ghia, U. 1989 Analysis of incompressible massively separated viscous flows using unsteady Navier-Stokes equations. Intl J. Numer. Meth. Fluids 9, 10251050

Goldstein, R. J., Eriksen, V. L., Olson, R. M. \& Eckert, E. R. G. 1970 Laminar separation, reattachment, and transition of the flow over a downstream-facing step. Trans. ASME D: J. Basic Engng 92, 732-739. 
Gresho, P. M., Gartling, D. K., Torczynski, J. R., Cliffe, K. A., Winters, K. H., Garratt, T. J., SPENCE, A. \& Goodrich, J. W. 1993 Is the steady viscous incompressible two-dimensional flow over a backward-facing step at Re $=800$ stable? Intl J. Numer. Meth. Fluids 17, 501-541.

Hamilton, J. M., Kim, J. \& WalefFe, F. 1995 Regeneration mechanisms of near-wall turbulence structures. J. Fluid Mech. 287, 317-348.

Hammond, D. A. \& Redekopp, L. G. 1998 Local and global instability properties of separation bubbles. Eur. J. Mech. B/Fluids 17, 145-164.

Henderson, R. D. 1994 Unstructured spectral element methods: parallel algorithms and simulations. $\mathrm{PhD}$ thesis, Princeton University.

HENDERSON, R. D. \& KARNiADAKIs, G. E. 1995 Unstructured spectral element methods for simulation of turbulent flows. J. Comput. Phys. 122, 191-217.

Huerre, P. \& Monkewitz, P. A. 1990 Local and global instabilities in spatially developing flows. Annu. Rev. Fluid Mech. 22, 473-537.

KaikTsis, L., Karniadakis, G. E. \& OrszaG, S. A. 1991 Onset of three-dimensionality, equilibria, and early transition in flow over a backward-facing step. J. Fluid Mech. 231, 501-528.

Kaiktsis, L., Karniadakis, G. E. \& Orszag, S. A. 1996 Unsteadiness and convective instabilities in two-dimensional flow over a backward-facing step. J. Fluid Mech. 321, 157-187.

Karniadakis, G. E., Israeli, M. \& Orszag, S. A. 1991 High-order splitting methods for the incompressible Navier-Stokes equations. J. Comput. Phys. 97, 414-443.

LE, H., MoIn, P. \& KIM, J. 1997 Direct numerical simulation of turbulent flow over a backward-facing step. J. Fluid Mech. 330, 349-374.

Mamun, C. K. \& Tuckerman, L. S. 1995 Asymmetry and Hopf bifurcation in spherical Couette flow. Phys. Fluids 7, 80-91.

Patera, A. T. 1984 A spectral element method for fluid dynamics: laminar flow in a channel expansion. J. Comput. Phys. 54, 468-488.

SAAD, Y. \& Schultz, M. H. 1986 Gmres-a generalized minimal residual algorithm for solving nonsymmetric linear-systems. SIAM J. Sci. Statist. Comput. 7, 856-869.

Schatz, M. F., Barkley, D. \& Swinney, H. L. 1995 Instabilities in spatially periodic channel flow. Phys. Fluids 7, 344-358.

Williams, P. T. \& BAKER, A. J. 1997 Numerical simulations of laminar flow over a three-dimensional backward-facing step. Intl J. Numer. Meth. Fluids 24, 1159-1183. 